\title{
Análisis y caracterización fotométrica de un espacio de transición iluminado con luz natural: sus implicancias en la visión funcional
}

\author{
Photometric analysis and characterization of a transition \\ space illuminated with daylight: its implications for \\ functional vision
}

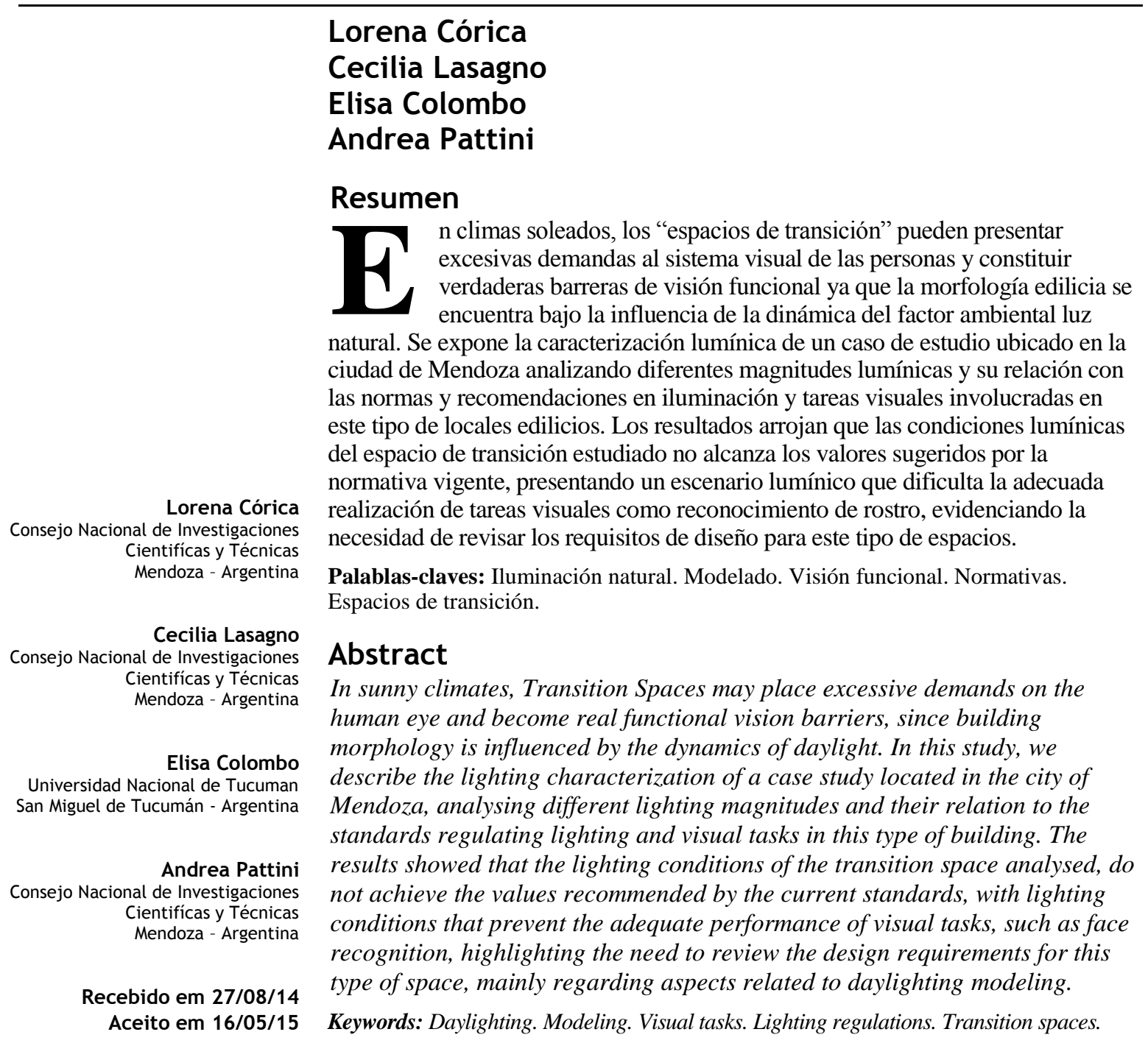

CÓRICA, L.; LASAGNO, C.; COLOMBO, E.; PATTINI, A. Análisis y caracterización fotométrica de un espacio de transición iluminado con luz natural: sus implicancias en la visión funcional. Ambiente Construído, Porto Alegre, v. 15, n. 3, p. 103-115, jul./set. 2015. 


\section{Introducción}

La variedad de espacios de transición del ambiente construido da lugar a la generación de muy diversas situaciones de uso, que pueden complejizarse si se consideran las características visuales de los usuarios y el clima luminoso de cada región donde están emplazados. En climas soleados, estos espacios constituyen un caso paradigmático, ya que pueden presentar excesivas demandas al sistema visual y constituir verdaderas "barreras de visión funcional" (COLOMBO et al., 2013; GINSBURG, 2003). Estas barreras, hacen referencia a aquellos factores del ambiente iluminado que pueden dificultar o impedir la tarea visual, causando un mal desempeño del mismo.

En general, un espacio de transición se define como un "espacio independiente que posee variadas condiciones físicas y comportamentales" (CHUN; KWOK; TAMURA, 2004), las cuales pueden estar influenciadas en el período de uso diurno por la dinámica de la luz natural. Los marcados cambios de luminancias que experimentan estos escenarios impiden que el proceso de adaptación visual del usuario se complete en el tiempo necesario para atravesarlos, especialmente cuando la transición se realiza del exterior al interior del edificio y viceversa. Junto a esto, aparecen otros efectos no deseados como deslumbramiento o reflejos especulares, que se traducen en molestias visuales (disconfort) o disminución de la eficiencia visual.

Por otro lado, en climas soleados se involucran variables no sólo del tipo edilicio, como la orientación de los espacios, la forma y características ópticas de los materiales, sino también variables propias de la dinámica del factor ambiental de la luz solar, a partir de las trayectorias solares, sus ángulos de incidencia y los efectos que las distintas componentes (directa, difusa y reflejada) ejercen en el medioambiente visual (FONTOYNONT, 1999; PATTINI, 2007; CÓRICA, 2010; GUZOWSKI, 2000). Como ya se dijo, este tipo de espacios se presentan como una potencial restricción para los usuarios, por lo que se debe tener en cuenta requisitos de diseño apropiados que contemplen aspectos de adaptación visual, a partir del diseño de la morfología de los mismos. La calidad de la iluminación natural está dada no solamente por la provisión de una buena cantidad de luz, sino también por los factores que son componentes del confort y de la eficiencia visual tales como: control del deslumbramiento, graduación de la luminancia, reducción de contrastes extremos, carácter direccional de la luz.

Desde el punto de vista lumínico, las recomendaciones de niveles de iluminación y relaciones para este tipo de espacios, con aporte de luz natural, no han sido desarrolladas en profundidad. Los códigos urbanos y normativas brindan acotadas recomendaciones basadas en iluminancias horizontales y verticales, como es el caso de Normas nacionales IRAM AADL J20-02 (INSTITUTO..., 1970) y J20-06 (INSTITUTO..., 1972) o como la norma CIE de carácter internacional (INTERNATIONAL..., 1992). En los espacios de transición se realizan principalmente tareas de detección y reconocimiento de objetos tridimensionales y en particular de rostros, es decir la percepción de modelado (sombras y profundidades). Los estudios de modelado desarrollados hasta el momento se centran principalmente en iluminación artificial nocturna (ROMBAUTS; VANDEWYNGAERDE; MAGGETTO, 1989; ROMBAUTS, 1995) obteniéndose índices con límites de iluminación mínimos que garanticen el reconocimiento de las personas y objetos. No obstante, se observa que las actividades cotidianas diurnas también presentan situaciones relacionadas con la iluminación que deberían ser reglamentadas o al menos contempladas dentro de normas de edificación (al menos en lugares de uso público) que aseguren la ausencia de barreras de visión funcional.

\section{Marco normativo}

Para poder evaluar la iluminación ya sea natural o artificial de espacios, se deben tener en cuenta los niveles recomendados para la actividad visual a desarrollar, para asegurar la realización de las tareas con eficacia y confort (PATTINI, 2000). Existen diferentes normativas y requisitos en aspectos luminotécnicos en ámbitos nacionales e internacionales para iluminación de espacios interiores como así también de iluminación urbana. En la mayoría de los casos, los desarrollos han profundizado el campo de la iluminación artificial según el estudio de diferentes magnitudes lumínicas en función del tipo de tarea:

(a) en Argentina, la Norma de iluminación nacional vigente IRAM AADL J20-06 establece valores mínimos para más de 200 actividades, clasificadas por tipo de edificio, local y tarea visual (INSTITUTO..., 1972, 1970). La misma contempla el parámetro de Iluminancia Horizontal (Eh) para determinar la cantidad de iluminación artificial necesaria, considerando la dificultad de la tarea que se llevará a cabo en cada local. Dentro de esta clasificación, los espacios de transición están enmarcados conforme al tipo de tarea visual en la categoría "solamente visión ocasional", con una exigencia de Eh mín: 100 lux. Y en la categoría 
"hall de entrada" de acuerdo al tipo de edificio, de premisas y de tarea visual, con una exigencia de Eh mín: 300 lux;

(b) en cuanto a normativas internacionales, la Illuminating Enginieering Society of North America (IESNA) publica el Lighting Handbook con lineamientos y propuestas a partir de los aportes de la comunidad luminotécnica internacional sobre los últimos avances en la temática (ILLUMINATING..., 2000).

Fundamentalmente establece rangos de iluminación obedeciendo a criterios cuantitativos y cualitativos más amplios, como uniformidad de iluminación, aspectos energéticos, prevención del deslumbramiento, etc. En la última edición del 2010, a partir de nuevos enfoques y procedimientos la IESNA recomienda valores mínimos de iluminancia horizontal e iluminancia vertical para la iluminación del hall de entrada en edificios (Tabla 1) e incorpora las variables "edad e intensidad de uso", no haciendo referencia a valores de iluminancias máximas aconsejadas (DILAURA et al., 2011); y

(c) la iluminación, tanto la artificial como la natural, afecta la habilidad de las personas para adquirir información visual del entorno, destacándose en particular el reconocimiento de rostros de las personas como una tarea asociada a parámetros de seguridad, confort y eficiencia. Para la evaluación de modelado de rostros y espacios, diversos estudios han demostrado que la relación entre la Ev/Esc brinda una buena guía en correspondencia al modelado (ROMBAUTS; VANDEWYNGAERDE; MAGGETTO, 1989; ROMBAUTS, 1995, 2001; WILDE; MANZANO, 1989). Dicha relación es considerada un parámetro suplementario de la iluminancia horizontal (Eh) para asegurar el reconocimiento facial (INTERNATIONAL..., 1992). En la mayoría de los casos, los desarrollos han profundizado en el campo de iluminación artificial.

El "modelado" se relaciona con el reconocimiento tridimensional y la sensación de agrado y aceptación de una instalación que puede ser juzgada sobre la naturalidad de la apariencia de la gente, además de la detección de objetos (INTERNATIONAL..., 1992).

Los contrastes no deberían ser ni excesivos ni escasos de manera de no distorsionar los rasgos de las personas y de la arquitectura. La dirección de la luz y la altura desde la que incide tiene una importancia decisiva en el aspecto general del reconocimiento. Variando la posición de la fuente, pueden resaltarse los detalles principales y ocultarse otros. De la dirección de la luz también depende la sensación de volumen, la textura y la intensidad de los colores, conduciendo a tres tipos de modelado:
(a) modelado duro: se presenta cuando el volumen muestra un excesivo contraste, dado por una fuerte direccionalidad de la luz que provoca sombras muy oscuras (duras) sobre el rostro;

(b) modelado plano/blando: se presenta cuando el volumen del rostro muestra mínimos contrastes, generado por un predominio de luz difusa, mostrándose aplanado y chato; y

(c) modelado natural: se presenta cuando el volumen del rostro muestra un contraste equilibrado y agradable, es posible observar que se trata de un volumen tridimensional, con detalles y texturas.

Para estos índices, se define como "modelado natural" al cociente entre Ev e Esc comprendido entre 0.8 y 1.5 ; mientras que los valores más cercanos al 0 corresponderían a modelados duros y los superiores a 1.5 a modelado blando (Tabla 2).

A partir de lo expuesto, se presenta la caracterización y estudio del comportamiento lumínico de un espacio de transición exteriorinterior ubicado en la ciudad de Mendoza como caso de estudio de climas soleados. El objetivo apunta a evaluar aspectos relacionados con la morfología construida y la dinámica de la luz natural en un período diurno. El abordaje se realiza considerando las recomendaciones y normativas vigentes que regulan el uso o la actividad en este tipo de locales edilicios.

Tabla 1 - Valores recomendados por la IESNA para iluminación en hall de entrada de edificios

\begin{tabular}{l|c|c|c|c|c|c|c}
\hline \multirow{2}{*}{} & \multicolumn{3}{|c|}{ Eh[lux] } & \multicolumn{4}{c}{ Ev [lux] } \\
\cline { 3 - 8 } & & $<25$ años & $\mathbf{2 5 - 6 5}$ años & $>65$ años & $<25$ años & $\mathbf{2 5 - 6 5}$ años & $>$ 65 años \\
\hline \multirow{2}{*}{ Media actividad } & Día & 50 & 100 & 200 & 25 & 50 & 100 \\
& Noche & 25 & 50 & 100 & 15 & 30 & 60 \\
\hline
\end{tabular}

Tabla 2 - Índices de modelado

\begin{tabular}{c|c}
\hline \multicolumn{2}{c}{ Índices de Modelado natural } \\
\hline CIE (1992) & $0.8 \leq \mathrm{Ev} / \mathrm{Esc} \leq 1.3$ \\
Rombauts (1989) & $1.1 \leq \mathrm{Ev} / \mathrm{Esc} \leq 1.5$ \\
\hline
\end{tabular}




\section{Metodología}

\section{Caracterización morfológica del espacio}

Para el presente estudio se toma un espacio de transición ubicado en el Centro Científico Tecnológico (CCT) - MENDOZA CONICET, ciudad de Mendoza, en el centro oeste de la Argentina. El territorio provincial se encuentra inserto en un clima semiárido, presentando un alto porcentaje de cielos claros a lo largo del año. Como valores representativos se registran disponibilidades de luz natural elevadas según las condicionantes ambientales del clima regional correspondiente a cielos soleados (Iluminancia horizonatal global: 120000lx en verano; 65000lx en invierno) según datos registrados por el Servicio Meteorológico Nacional Argentina. 2001-2010.

Figura 1 - Planimetría del espacio

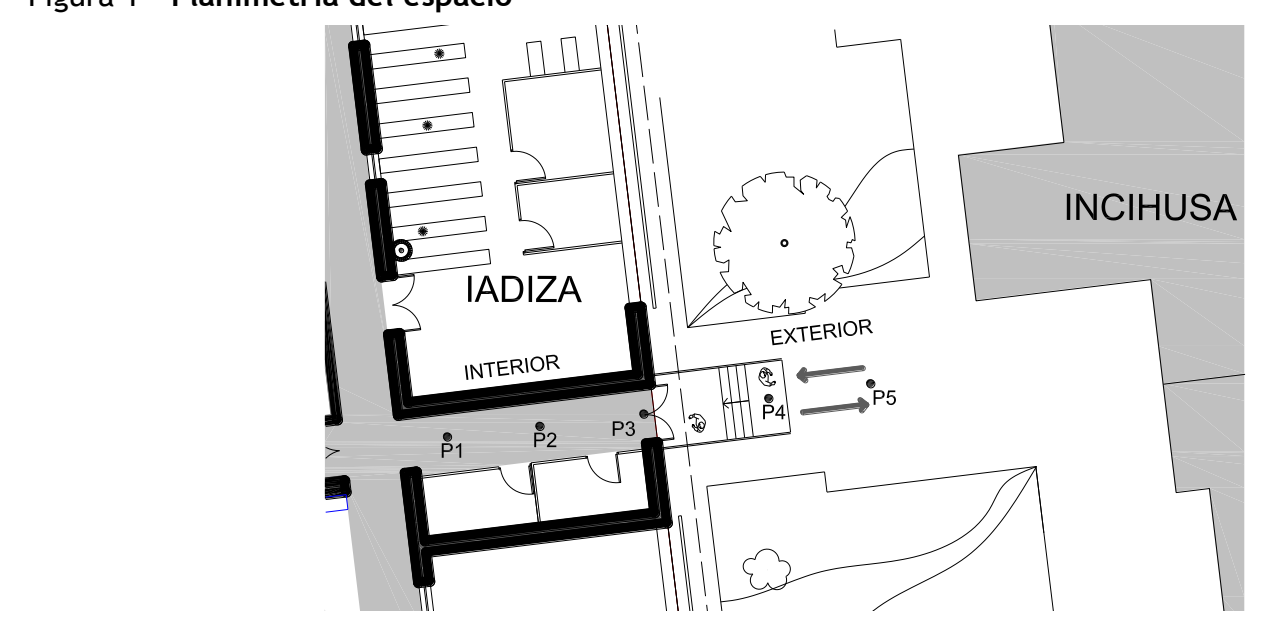

Figura 2 - Visión de cielo del entorno (ojo de pez)

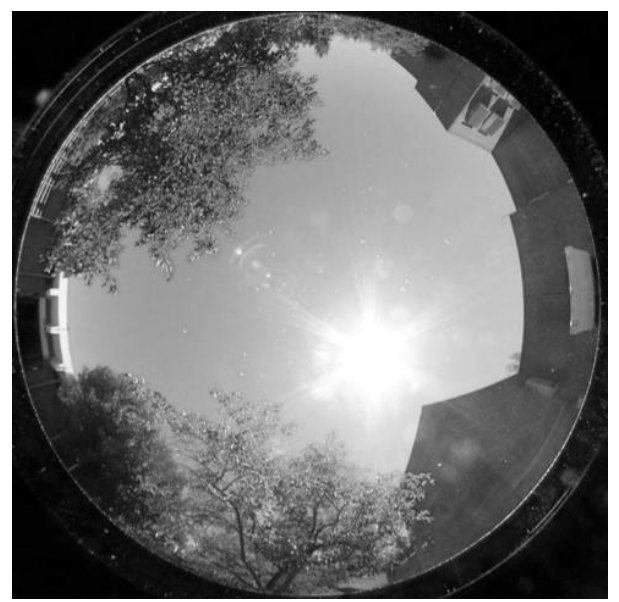

106 Córica, L.; Lasagno, C.; Colombo, E.; Pattini, A.
El caso de estudio se encuentra en un sector conformado por un espacio abierto con circulaciones peatonales y áreas verdes y acceso al edificio construido del centro de investigación. Como lo muestra la Figura 1, el mismo está definido por el área interior del edificio IADIZA (con un nivel de altura), el espacio exterior de jardines y por el edificio INCIHUSA (dos niveles de altura), ubicado en el frente este, como se observa en la imagen de visión de cielo, tomada en el punto 5 (Figuras 1 y 2). El área de intervención corresponde al ingreso sur del edificio IADIZA, con un trayecto interior de $6 \mathrm{~m}$ y espacio exterior conexo de $12 \mathrm{~m}$ aproximadamente hasta el edificio enfrentado. La orientación del trayecto, se apoya sobre sentido de eje Este-Oeste.

Este espacio de transición presenta una geometría de acceso frontal y directo desde el exterior al interior, sin dispositivos de control o adaptación previa, es decir sin presencia de pérgolas o filtros que permitan una adaptación visual paulatina. 
Desde el punto de vista morfológico, los acabados exponen materiales con reflectancias bajas en superficies de suelo (baldosa azul) y muros laterales (ladrillos y paneles beige oscuro), mientras que el techo es claro con heterogeneidad por casetonados. El aporte de luz natural en el interior, proviene de la doble puerta de acceso vidriada con orientación al este, por lo que en horas del día recibe luz directa y difusa en su gran mayoría.

En cuanto al espacio exterior, la morfología del entorno, se presenta como un espacio abierto. La superficie horizontal consiste en una explanada con acabados claros (gris) y sectores de verdes con presencia de forestales. Los muros de los edificios que delimitan el sector presentan reflectancias medias (ladrillos claros por envejecimiento de color en el exterior).

\section{Relevamiento fotométrico del espacio}

La evaluación lumínica estuvo organizada sobre el relevamiento fotométrico del espacio de desplazamiento de una persona sobre un eje lineal en la transición interior- exterior. Para un análisis detallado se establecieron puntos de medición (P), distribuidos a lo largo del trayecto y a una distancia equidistante de $3 \mathrm{~m}$. Se definieron P1 y $\mathrm{P} 2$ en el interior del hall, P3 en el umbral (sector de puerta de ingreso) y P4 - P5 en el exterior (Figura 3). En este sentido, se establecieron dos condiciones, teniendo en cuenta los usos del mismo: Condición de desplazamiento Caso A Ingreso al edificio y Caso B - Egreso del edificio.
Se realizaron mediciones para la condición de verano, como situación crítica del año al presentar los máximos valores de iluminancias en el exterior, para una jornada completa (11hs a 16hs). Con el objeto de calcular los índices existentes que podrían caracterizar este espacio de transición se relevaron diferentes parámetros: registros de Iluminancia Horizontal (Eh), Iluminancia Vertical (Ev), Iluminancia Semicilíndrica (Esc) e Iluminancia Cilíndrica (Ec). Esta exhaustiva etapa de monitoreo permitió obtener un volumen de registros significativos para describir fotométricamente el espacio y observar las condiciones lumínicas a las que se someten las personas en función de la dinámica de la luz natural (variaciones y direccionalidad de la fuente por trayectoria solar) y de las componentes de la misma según las características morfológicas de la edilicia y del entorno construido.

Los equipos utilizados fueron dos luxímetros: un radiómetro LI-COR 189 con sensor fotométrico LI-210 SB para el registro de Iluminancia horizontal y vertical y un Luxímetro marca LMT con cabezal para medición de iluminancia cilíndrica de rango 0.1 a 199.990 lux.

Los sensores fueron ubicados a una altura de $1.50 \mathrm{~m}$. La toma fotográfica fue realizada con cámara digital Nikon Colpix 5400 a una distancia de $3 \mathrm{~m}$ entre el instrumental y la modelo, identificada como "zona social", distancia mínima a la cual sería esperable reconocer la persona que circula en sentido contrario (Caminada Van Bommel, 1984). Para el cálculo de de iluminancia semi-cilíndrica, se realizaron mediciones de Ev cada $45^{\circ}$ de rotación en un ángulo de giro de $180^{\circ}$.

Figura 3 - Vista de los puntos establecidos en el interior y el exterior
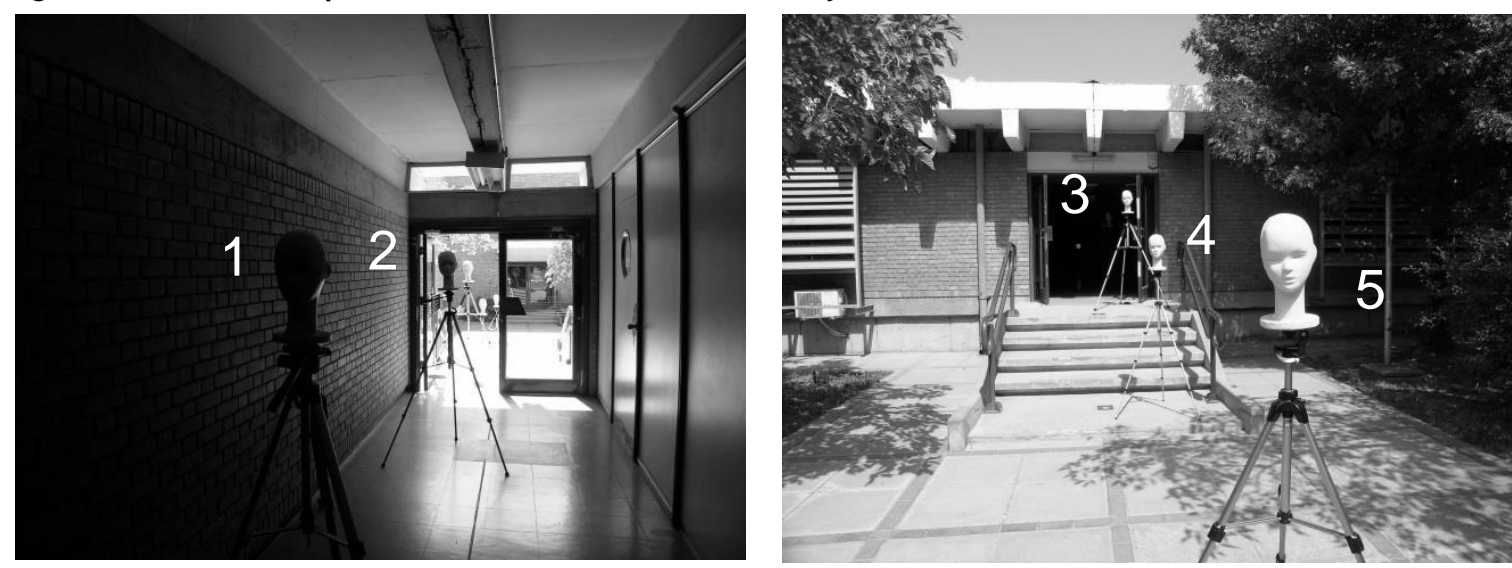


\section{Resultados}

\section{Iluminancia horizontal}

Los resultados obtenidos para la toma de Eh exhibidos en la Figura 4, muestran de manera clara la secuencia de diferentes rangos lumínicos en el período diurno, para el recorrido entre el espacio exterior-interior. Los valores expresan una gran amplitud que oscila entre los $100 \mathrm{~lx}$ en el interior y 120000 lx en el exterior, en un período de tiempo comprendido entre las $11 \mathrm{hs}$ y las $16 \mathrm{hs}$.

Estas grandes variaciones de iluminancia obtenidas para el relevamiento fotométrico presente, responden a consideraciones geométricas y morfológicas que modifican la disponibilidad lumínica a partir de su relación con la luz natural. En el espacio interior, las características dimensionales a partir de un espacio estrecho y con profundidad en el sentido de proporciones largoancho- alto (entrada tipo túnel), sumado al bajo porcentaje de abertura y la mala orientación, hacen que el aporte de luz natural disminuya drásticamente en relación al medioambiente lumínico exterior. Los colores y el tipo de acabado de las superficies tienen una repercusión directa. Se trata de colores oscuros con bajo coeficiente de reflexión, produciendo la disminución en los valores de iluminancias además de una falta de uniformidad en la distribución lumínica.

Por el contrario, en la zona exterior, los valores aparecen elevados dadas las intensidades luminosas regionales descriptas. Las características arquitectónicas y morfológicas del espacio abierto y las propiedades superficiales de altas reflectancias, hacen que además de la incidencia de luz directa, se potencie la componente reflejada exterior no sólo proveniente de los muros colindantes verticales sino también la luz reflejada por el piso. Esto se evidencia fundamentalmente a partir del mediodía solar.

Por otro lado, si se centra el análisis en los registros de cada hora, puede notarse la influencia que ejerce la direccionalidad de la luz natural, no sólo a partir de la dinámica solar diurna sino también en el efecto que ejercen las componentes en el entorno construido, a partir de la localización del espacio con eje de orientación en sentido esteoeste.

Como ejemplo de esta afirmación, los puntos medidos en el interior del edificio en el período matutino (PUNTOS 1; 2; 3), arrojan los máximos rangos lumínicos alcanzados durante el día. La fachada del edificio recibe luz directa en este período de la jornada permitiendo la penetración de sol directo dentro del espacio interior y en consecuencia favoreciendo el aporte de la luz reflejada emitida por las superficies del hall (11hsP1:200 1x; P2: 890 1x). Pero, con el transcurso de las horas y en función de la rotación de los ángulos solares, los valores en dichos puntos van decreciendo, arrojando las iluminancias más bajas en las últimas horas de la sesión de la tarde (16hsP1:50 lx; P2: 115 lx). En los puntos registrados en el exterior, en general se presentan valores elevados $\quad(97000 \quad 1 x \quad$ a $\quad 117000 \quad$ lx aproximadamente), registros correspondientes con las intensidades emitidas por la iluminancia horizontal global según los ángulos de altitud solar.

Figura 4 - Gráfico de lluminancias horizontales para el período diurno

lluminancias Horizontales en el espacio

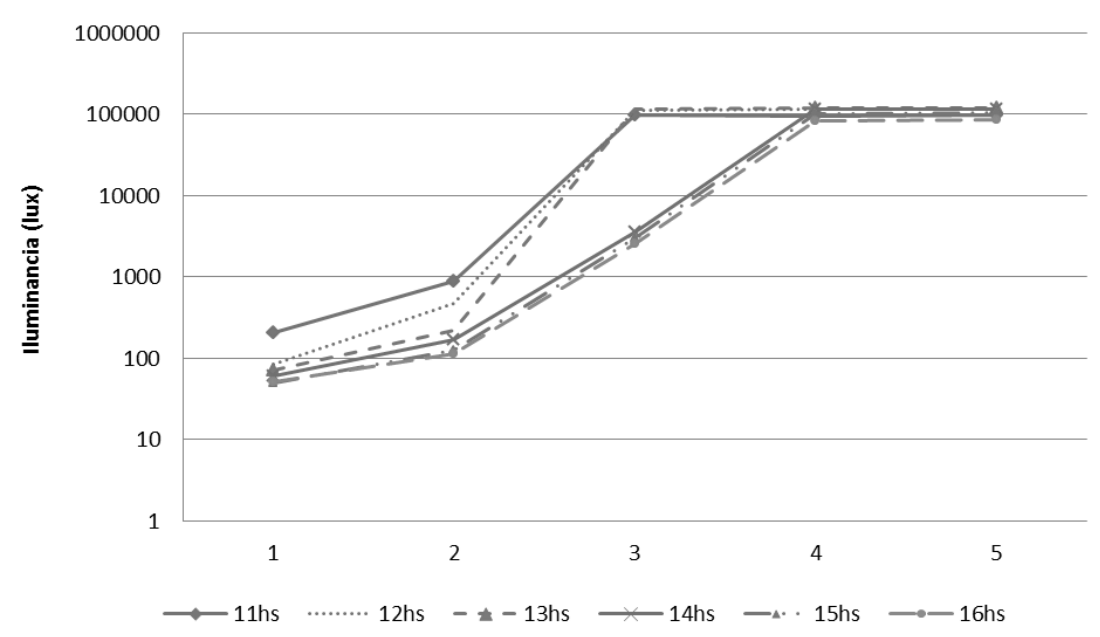

108 Córica, L.; Lasagno, C.; Colombo, E.; Pattini, A. 
Cabe aclarar que en el período vespertino, se produce un efecto lumínico particular, precisamente entre las $15 \mathrm{hs}$ y las $16 \mathrm{hs}$, donde más allá de la pérdida de intensidad de la fuente por la altitud solar, no se observa gran variación entre las iluminancias de todos los puntos registrados para ambos horarios $y$ este efecto responde fundamentalmente al aporte de la componente reflejada proveniente del edificio enfrentado que delimita la morfología del espacio exterior.

Pero, más allá de esta la contribución de luz reflejada, si se tiene en cuenta la normativa, en el interior del espacio (puntos 1 y 2) se registran los valores más desfavorables de la jornada. La profundidad y geometría del espacio, hacen que a medida que los puntos medidos se alejan de la abertura reduzcan sus registros, resultando inferiores a 3001x. Esto, además de estar fuera de las recomendaciones resulta un despropósito si se consideran las intensidades luminosas del entorno exterior. Por lo que sería posible pensar en un mejoramiento lumínico mediante el diseño de sistemas de iluminación natural que optimicen las condiciones visuales. Todo esto cobra mayor importancia si se considera el intensivo uso diurno de algunos edificios y la disponibilidad global del clima luminoso regional.

\section{Evaluación iluminancias vertical y semicilíndrica}

Como variables influyentes para la realización de las tareas visuales de reconocimiento de rostro y del espacio, se obtuvieron datos de Iluminancia vertical $(E v)$ e iluminancia semicilíndrica $(E s c)$.

Las Figura 5 y 6 y, Tablas 3 y 4 exponen los resultados del monitoreo para los recorridos de entrada y salida en cada punto de análisis. Al igual que en los registros de iluminancia horizontal, las grandes variaciones de rangos entre el espacio abierto y el construido son una constante, no sólo entre los puntos medidos a cada hora sino también en las alternancias que se dan a lo largo de la jornada.
Para la condición de ingreso (Caso A) cuando se transita en el espacio abierto para acceder al hall, durante horas de la mañana las personas se exponen a condiciones de luz difusa, ya que el sol se encuentra ubicado por detrás de su campo visual. Si bien no se presentan deslumbramientos, los sujetos se encuentran adaptados a niveles de iluminación altos (10000 lx) comparados a los que dispone el interior $(50 \mathrm{~lx})$, generando un marcado cambio en la luminancia de adaptación en un lapso de tiempo muy breve. Los cambios drásticos y repentinos en la iluminación, paso de la luz a la oscuridad o de la oscuridad a la luz, generan en el sistema visual pérdidas en su normal funcionamiento, con la posibilidad de provocar cegueras temporales. Esto conlleva a deslumbramientos discapacitantes y provoca un deterioro de las funciones visuales, causando la pérdida de la visibilidad debido al decrecimiento en el contraste de la imagen retiniana (VOS, 1984; ILLUMINATING..., 2000) o porque la escena se oscurece de manera abrupta. En horas de la tarde, los escenarios se invierten y esto nuevamente evidencia la direccionalidad de la fuente. A partir del mediodía solar, los sujetos que ingresan comienzan a recibir sobre su rostro la incidencia de luz directa debido a los ángulos de rotación solar y la ubicación de sol generándose situaciones de deslumbramiento que continúan dificultando la realización de las tareas visuales en el interior del espacio de transición. Los cambios experimentados van de 72000 lx (P5-16hs) a 22 lx (P1-16hs).

Para la condición de egreso, (Caso B) (Figura 6 y Tabla 4) todos los valores resultan superiores comparados al Caso A, superiores a los 100001x como consecuencia de la visión hacia el exterior que implica el sentido de desplazamiento. Las principales variaciones están determinadas por la direccionalidad de la luz natural y el efecto de la componente reflejada en el entorno construido, el cual se acentúa en horas de la tarde por el aumento en las luminancias y reflectancias superficiales provenientes del edificio enfrentado al espacio.

Figura 5 - Secuencia de recorrido para el ingreso al espacio

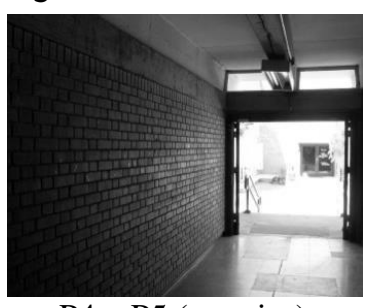

P4 - P5 (exterior)

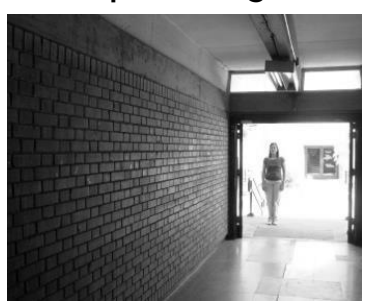

P3 (umbral del acceso)

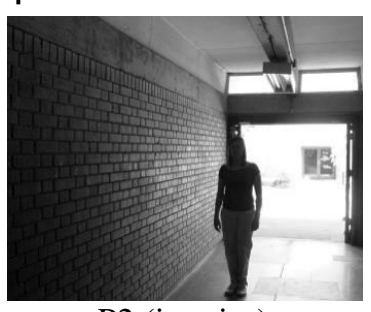

P2 (interior)

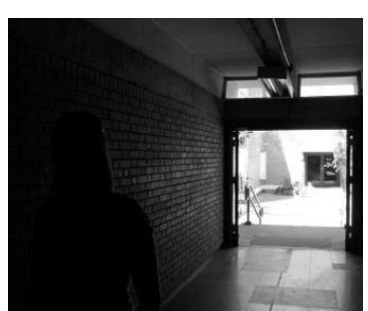

P1 (interior) 
Tabla 3 - Valores obtenidos para Ev y Esc en condición de Ingreso

\begin{tabular}{c|c|c|c|c|c|c|c|c|c|c|c|c}
\hline \multirow{2}{*}{ Ingreso } & \multicolumn{2}{|c|}{$\mathbf{1 1 h}$} & \multicolumn{2}{c|}{ 12hs } & \multicolumn{2}{c|}{ 13hs } & \multicolumn{2}{c|}{ 14hs } & \multicolumn{2}{c|}{ 15hs } & \multicolumn{2}{c}{ 16hs } \\
\cline { 2 - 13 } & $\boldsymbol{E} \boldsymbol{v}$ & $\boldsymbol{E} \boldsymbol{c} \boldsymbol{E}$ & $\boldsymbol{E} \boldsymbol{v}$ & $\boldsymbol{E} \boldsymbol{s}$ & $\boldsymbol{E} \boldsymbol{v}$ & $\boldsymbol{E} \boldsymbol{c}$ & $\boldsymbol{E} \boldsymbol{v}$ & $\boldsymbol{E} \boldsymbol{c}$ & $\boldsymbol{E} \boldsymbol{v}$ & $\boldsymbol{E} \boldsymbol{c}$ & $\boldsymbol{E} \boldsymbol{v}$ & $\boldsymbol{E} \boldsymbol{c}$ \\
\hline $\mathbf{1}$ & $\mathbf{5 0 , 8}$ & 59,4 & $\mathbf{2 9 , 9}$ & 27 & $\mathbf{2 7}$ & 23,8 & $\mathbf{2 4 , 1}$ & 20,9 & $\mathbf{2 0}$ & 20,5 & $\mathbf{2 1 , 9}$ & 18,23 \\
$\mathbf{2}$ & 206 & 304 & 108,7 & 143,1 & $\mathbf{5 8 , 6}$ & 62,6 & $\mathbf{5 2 , 1}$ & 57,6 & $\mathbf{4 9 , 4}$ & 48,5 & $\mathbf{4 5 , 8}$ & 44,9 \\
$\mathbf{3}$ & 4150 & 6090 & 2750 & 6630 & 2040 & 6620 & 530 & 1027 & 536,2 & 895 & 379,2 & 638 \\
$\mathbf{4}$ & 8390 & 6870 & 8820 & 10260 & 6490 & 10020 & 36780 & 19450 & 48160 & 26700 & 71740 & 3280 \\
$\mathbf{5}$ & 9130 & 8100 & 8560 & 21200 & 8150 & 10840 & 34120 & 20800 & 59800 & 27800 & 72560 & 35300 \\
\hline
\end{tabular}

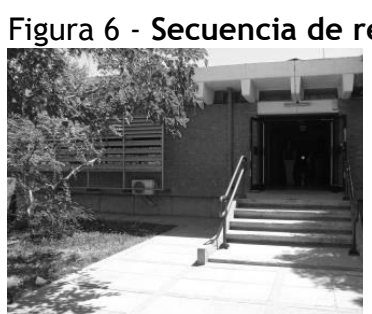

P1- P2 (interior)

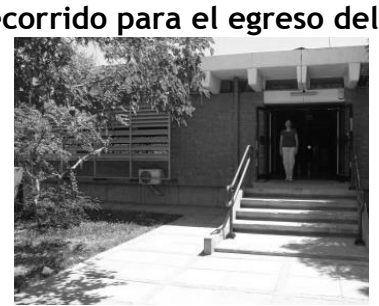

P3 (umbral del acceso)

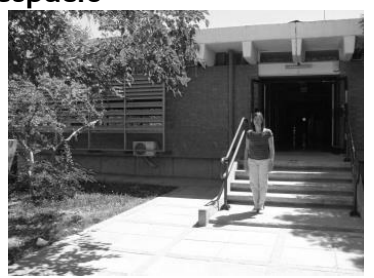

P4 (exterior)

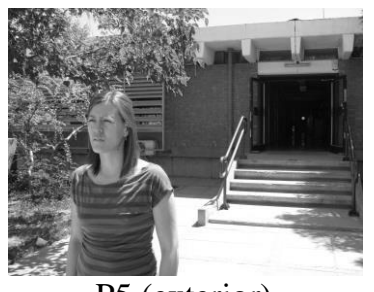

P5 (exterior)

Tabla 4 - Valores obtenidos para Ev y Esc en condición de Egreso

\begin{tabular}{|c|c|c|c|c|c|c|c|c|c|c|c|c|}
\hline \multirow{2}{*}{ Egreso } & \multicolumn{2}{|c|}{$11 \mathrm{hs}$} & \multicolumn{2}{|c|}{$12 \mathrm{hs}$} & \multicolumn{2}{|c|}{ 13hs } & \multicolumn{2}{|c|}{$14 \mathrm{hs}$} & \multicolumn{2}{|c|}{$15 \mathrm{hs}$} & \multicolumn{2}{|c|}{$16 \mathrm{hs}$} \\
\hline & $E v$ & $E s c$ & $E v$ & Esc & $E v$ & Esc & $E v$ & Esc & $E v$ & $E s c$ & $E v$ & Esc \\
\hline 1 & 729,7 & 3553 & 351,9 & 159,9 & 306,5 & 145,3 & 312,4 & 130,5 & 330 & 135,7 & 364,5 & 137,6 \\
\hline 2 & 2620 & 1417 & & & 793,7 & & & & 769,9 & & 826,3 & 295 \\
\hline 3 & 70280 & 32400 & 39050 & 20100 & 15420 & 13700 & 7340 & 4470 & 6150 & 3580 & 5070 & 2990 \\
\hline 4 & 69630 & 30800 & 40210 & 20400 & 14530 & 141900 & 10810 & 10790 & 9900 & 9960 & 8690 & 6790 \\
\hline 5 & 70110 & 31300 & 31230 & 9470 & 15030 & 14870 & 13280 & 12180 & 13500 & 10130 & 11650 & 8810 \\
\hline
\end{tabular}

Las curvas de la Figura 7 muestran los contrastes lumínicos registrados en la jornada, con variaciones extremas que se dan en los rangos medidos durante la primera y última sesión monitoreada y para los dos casos analizados. En la mañana las iluminancias no presentan cambios drásticos en el acceso al edificio (luz difusa sobre el campo visual), pero no así en el egreso, ya que los usuarios reciben incidencia de luz solar directamente sobre su rostro (69630lx). A las 16hs esta condición se revierte ya que en el ingreso el cambio en el azimut solar genera luz directa sobre el rostro de las personas y luz difusa para el egreso, ya que la fuente se encuentra por detrás de los sujetos dada la orientación del espacio.

En relación a los valores recomendados en $E v$ por la IESNA, en general los registros superan ampliamente los valores sugeridos para el rango etario y para un espacio de transitabilidad media. Sólo para la situación de ingreso en los puntos interiores 1 y 2 , se obtienen valores por debajo de la mínima que no alcanzan los 50 lx propuestos.

\section{Modelado}

\section{Ingreso}

La Figura 8 grafica el cociente entre Ev e Esc registrados para el ingreso al espacio, sólo el $43 \%$ del total de las relaciones están dentro del rango de "modelado natural" propuesto por la CIE $(0.8 \leq$ Ev/Esc $\leq 1.3)$. Al examinar detalladamente el rostro fotografiado en cada uno de los puntos y según estas recomendaciones, en el interior del espacio el sujeto ubicado en $\mathrm{P} 1$, resulta con condiciones de "modelado natural" en todos los horarios, mientras que en P2 sólo a partir del mediodía. Esto es llamativo ya que en el interior del espacio predominan bajos valores de iluminancias, los cuales están fuera de Norma tanto para Eh como para Ev. Por consiguiente, si se observan las imágenes registradas (Figuras 9a y 8b. Modelados dentro del rango "modelado natural", CIE) los rostros se manifiestan planos, difusos y en sombra, con contrastes mínimos debido a la ausencia de luz lo cual dificulta la apreciación del volumen facial. Según los valores obtenidos para el punto 3 (bajo el umbral de la puerta), en ningún momento del día las relaciones se circunscriben al rango de modelado natural, resultando índices de modelado duro. Los puntos 4

110 Córica, L.; Lasagno, C.; Colombo, E.; Pattini, A. 
y 5 arrojan índices de modelados óptimos en horas de la mañana mientras que el resto de las imágenes registra datos cuyos índices arrojan modelados planos. Debe aclararse que para este caso de estudio de modelado con luz natural se producen dos situaciones que responden a lo que queda definido como modelado plano: por un lado, situaciones con predominio de luz difusa sobre el rostro que lo aplana, dificultando ver los detalles. Por otro lado, modelado de luz difusa por detrás del rostro, generando una condición de sombras que unifica todos los detalles y también lo aplana.

No obstante esto, según el análisis de las imágenes de la modelo obtenidas en el espacio, existen momentos del día en los cuales el reconocimiento tridimensional es agradable y con contrastes equilibrados, principalmente en la mañana y el mediodía. Ejemplo de esta afirmación se muestra en las imágenes de la Figura 10a y 10b. Pero en dichas escenas, el cociente de modelado según la CIE las clasifica como "modelado duro".

\section{Egreso}

La Figura 11 grafica el cociente entre Ev e Esc registrados para la salida del recinto donde sólo el $30 \%$ de los mismos se ajustan a los índices sugeridos (Figura 10). El 60\% restante, si bien no se encuentra dentro del parámetro de modelado natural, presenta casos con una buena lectura de las 3 dimensiones aunque su cociente clasifica dentro de "modelado plano", Figuras 12 y 13. En el interior del edificio, los dos puntos medidos se encuentran fuera de los límites durante la jornada completa. Para los puntos exteriores P4 y P5, los cocientes entran dentro del rango "modelado natural" desde las $13 \mathrm{hs}$ en adelante.

\section{Figura 7 - Influencia de la direccionalidad de la iluminación natural sobre las lluminancias Verticales del campo visual}

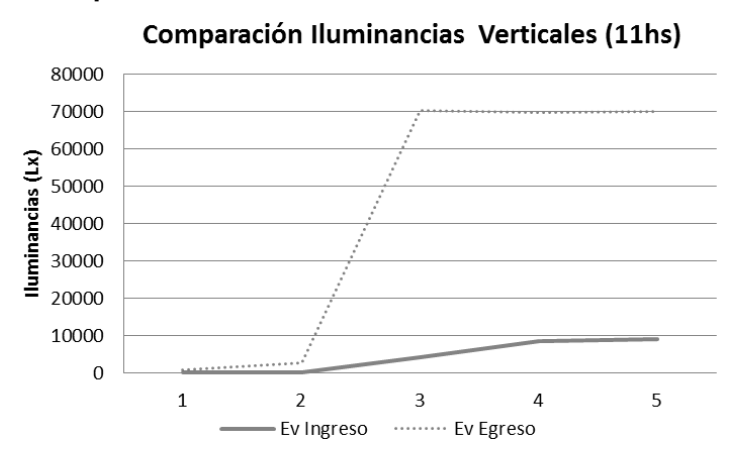

\section{Comparación Iluminancias Verticales (16hs)}

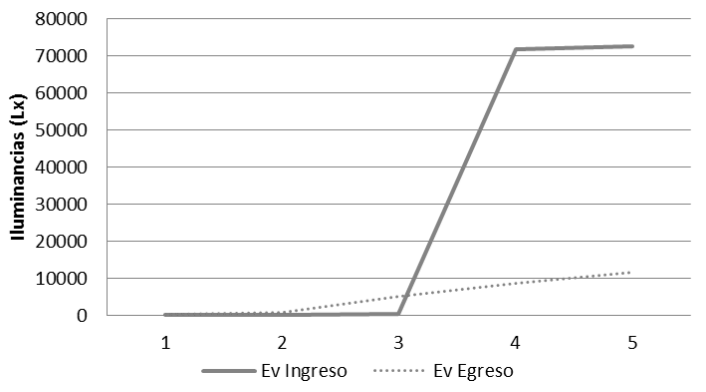

Figura 8 - Ingreso del edificio

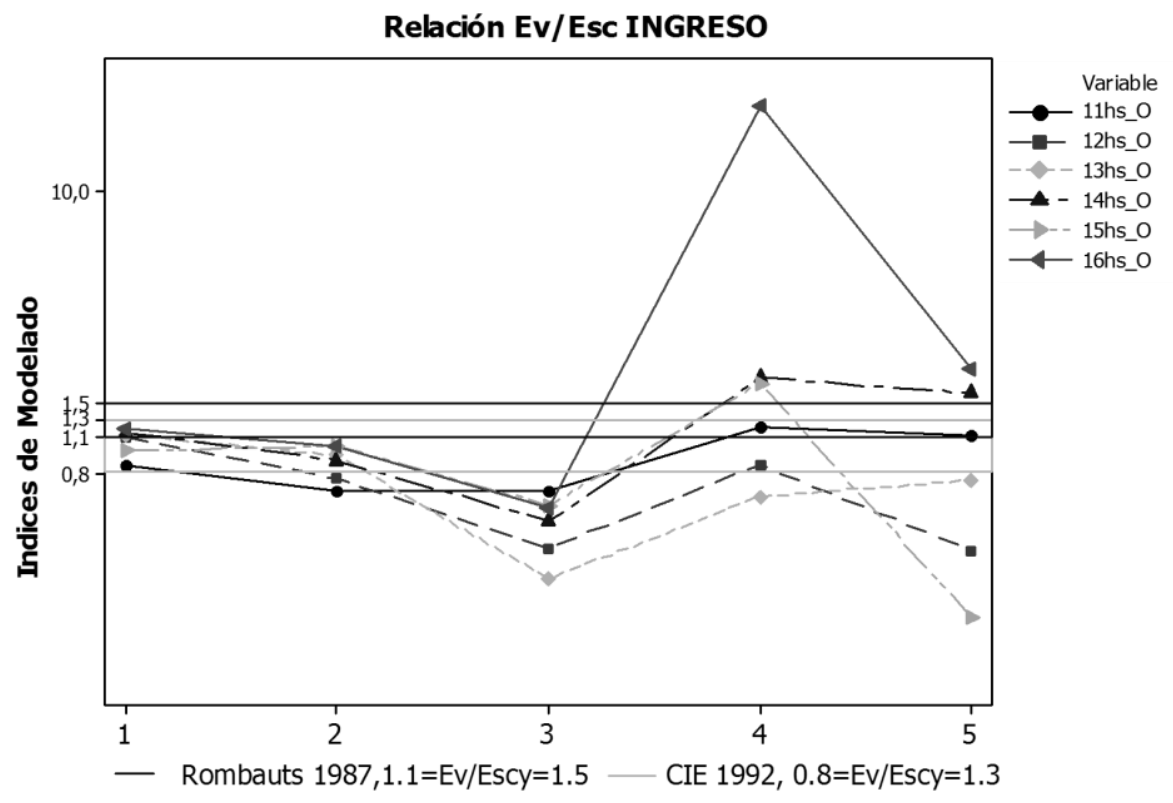


Figura 9 - Modelados dentro de rango CIE

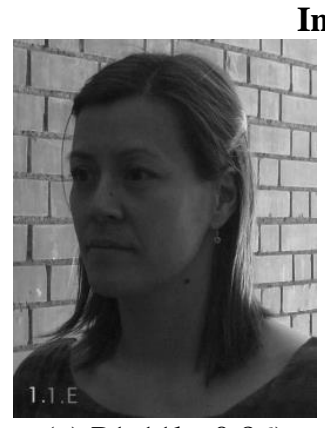

(a) P1-11hs 0.86)
Ingreso

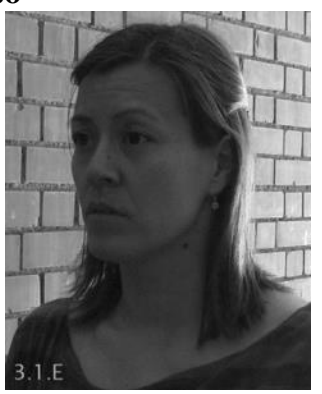

(b) P1- 13hs (1.13)

Figura 10 - Modelados fuera de rango CIE

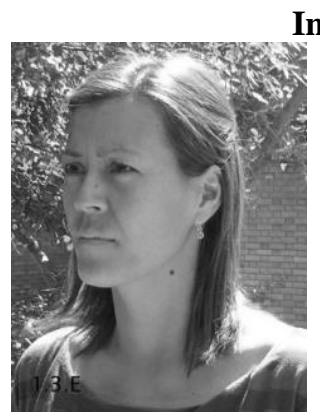

(a) P3- 12hs (0.41)
Ingreso

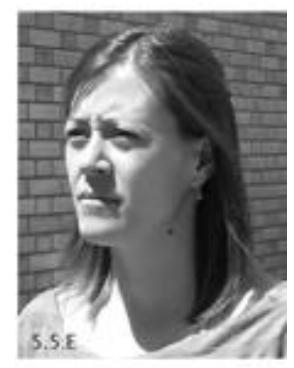

(b) $\mathrm{P} 5-15 \mathrm{hs}(0.22)$

Figura 11 - Egreso del edificio

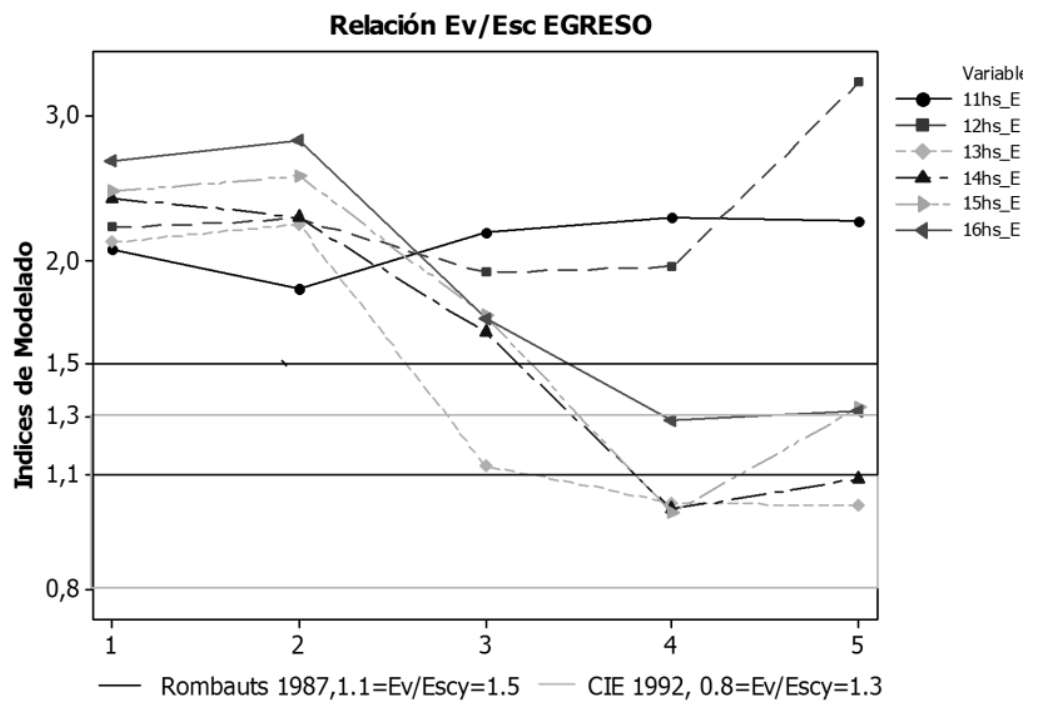

112 Córica, L.; Lasagno, C.; Colombo, E.; Pattini, A. 
Figura 12 - Modelados dentro de rango CIE

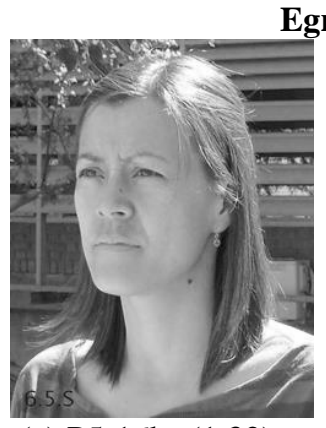

(a) P5-16hs (1.32)
Egreso

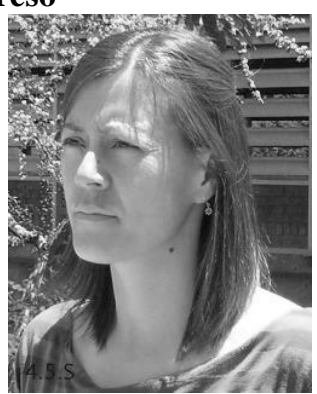

(b) P5- 14hs (1.09)

\section{Figura 13 - Modelados fuera de rango CIE}

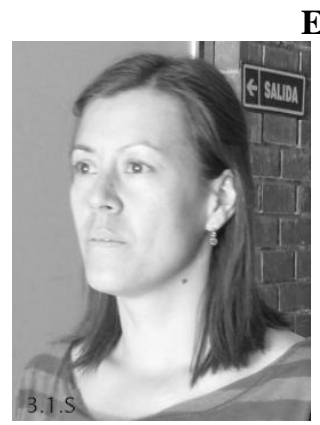

(a) P1- 13hs (2.11)

Egreso

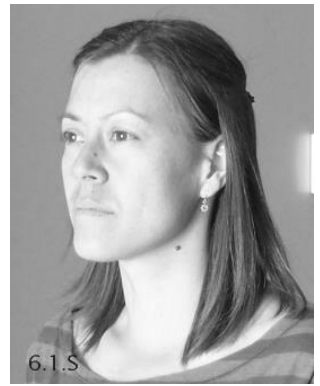

(b) P1-16hs (2.65)

\section{Conclusión}

Los resultados expuestos muestran un análisis detallado sobre el comportamiento y caracterización lumínica de un espacio de transición en climas soleados, a partir de una evaluación profunda desde distintas ópticas de estudio dentro de la luminotecnia. Dada la complejidad en el abordaje del caso planteado, surgen diferentes reflexiones a considerar: del relevamiento fotométrico de iluminancias horizontales para la tipología de espacio de transición analizada, se manifiestan grandes variaciones lumínicas entre el interior y el exterior. El estudio expone rangos muy bajos en el hall de entrada al edificio (P1 y P2) con una Eh promedio que no supera los 200 lux; mientras que en la zona del espacio abierto (P3 , P4 y P5) la Eh promedio es de 88000 lux. Este efecto antagónico obedece a la influencia que ejercen las características geométricas y morfológicas en las condiciones lumínicas. Aspectos de proporción (largo-ancho), dimensiones, orientación, sumados a los acabados superficiales y sus características ópticas repercuten en las distribuciones lumínicas del recorrido, principalmente en el espacio interior.

Estas condiciones generan excesivas demandas al sistema visual de las personas y pueden dificultar tareas visuales como: reconocimiento de rostro y

gestos, lectura de carteles orientativos, lecturas de tableros para control de ascensores, etc. Las recomendaciones lumínicas presentes en las normas y códigos urbanos, no contemplan requerimientos apropiados al carácter y uso de estas zonas, en particular cuando se trabaja en regiones con alta disponibilidad de luz solar. La situación descripta podría ser mitigada con un adecuado manejo de la iluminación para favorecer la "orientación visual", considerando la capacidad de la iluminación de transmitir información sobre el espacio:

(a) las condicionantes de la luz natural dadas por la direccionalidad de la fuente según la trayectoria y ángulos de altitud solar, como así también, las componentes de la luz (directa, difusa y reflejada), la orientación y distancia del espacio a recorrer, son variables que influyen en la manifestación del entorno construido, presentando diferentes escenarios lumínicos a lo largo del día. Por ende, no se puede subestimar el diseño de estos espacios, sino proyectarlos como ambientes dinámicos desde el punto de vista del factor ambiental luz solar, proponiendo diseños de Sistemas de Iluminación Natural que permitan graduar las diferencias de iluminación presentes entre el exterior e interior del mismo, creando zonas de media sombra para ir logrando la adaptación visual de las personas; 
(b) del estudio de modelado, se observa que las recomendaciones y los índices conocidos han sido elaborados para luz artificial y que según los análisis planteados no aplicarían a las características de la luz natural. La dirección e intensidad de la luz natural incidentes tienen una importancia decisiva en la representación de las 3 dimensiones. A partir de lo expuesto, surgen nuevos paradigmas de investigación a desarrollar. La mayoría de los antecedentes en la temática expuesta se han difundido para fuentes de luz artificial y para tareas visuales en espacios interiores, se pretende trabajar en estudios subjetivos sobre las preferencias de modelado de las personas según la dinámica de la luz natural en climas soleados. De esta forma, determinar nuevos índices adecuados a estas condicionantes ambientales; y

(c) las normativas y regulaciones analizadas, no contemplan la existencia de espacios de uso casi exclusivamente diurnos, es decir que reciben el aporte de luz natural directa y difusa durante la mayor parte del día con lo cual y debido a su gran intensidad modifica sustancialmente los niveles de iluminancia horizontal sugeridos. En este caso, los niveles de iluminación recomendados utilizan principalmente el parámetro de iluminancia horizontal, lo cual minimiza la complejidad del entorno construido en relación al aporte lumínico ocasionado por la reflexión que generan los materiales utilizados en la construcción, es decir la interacción entre la luz y las propiedades ópticas de los materiales (reflectancia).

Finalmente, a la hora de diseñar este tipo de espacios, es necesario realizar estudios previos en función de las respuestas que las conformaciones morfológicas van a ofrecer a los usuarios, según las disponibilidades globales de la iluminación regional y las particularidades del espacio construido, tales como: orientación del ingreso, tipo de tareas visual a realizar, reflectancias de los materiales intervinientes para un control de contrastes presentes en la escena, y demás variables arquitectónicas. De esta forma, garantizar una iluminación de calidad que favorezca el uso del espacio construido de modo seguro y en confort.

\section{Bibliografía}

CAMINADA, I. J. F.; VAN BOMMEL, K. W. J. M. New Lighting Criteria For Residential Areas. Journal of IES, 1984. Mais dados

CHUN, C.; KWOK, A.; TAMURA, A. Thermal Comfort in Transitional Spaces -basic concepts: literature review and trial measurement. Building and Environment, v. 39, p. 1187-1192, 2004.
COLOMBO, E. M. et al. Functional Vision Barriers: a new concept analyzed in terms of human visual performance. Psychology \& Neuroscience, v. 6, n. 2, p. 219-226, 2013.

CÓRICA, M. L. Comportamiento de la Luz Natural en Entornos Urbanos Representativos del Modelo Oasis en Regiones Áridas: caso de estudio, ciudad de Mendoza. 2010. Tesis (Doctorado) -Facultad de Ciencias Exactas y Tecnología, Universidad Nacional de Tucumán, Tucumán, 2010.

DILAURA, D. et al. The Lighting Handbook: reference and application. 10. ed. New York: Illuminating Engineering Society, 2011.

FONTOYNONT, M. Daylight Performance of Buildings. Paris: James \& James, 1999.

GINSBURG, A. P. Contrast Sensitivity and Functional Vision. International Ophthalmology Clinics, v. 43, n. 2, p. 5-15, 2003.

GUZOWSKI, M. Daylighting For Sustainable Design. New York: McGraw-Hill, 2000.

ILLUMINATING ENGINEERING SOCIETY OF NORTH AMERICA. The IESNA Lighting

Handbook: reference \& application. IESNA, 2000.

INTERNATIONAL COMMISSION ON

ILLUMINATION. Guide to the Lighting of

Urban Areas. CIE, 1992. Technical Report.

INSTITUTO ARGENTINO DE

RACIONALIZACIÓN DE MATERIALES.

IRAM-AADL J20-06: iluminación artificial de interiores: niveles de iluminación. Buenos Aires, 1972.

INSTITUTO ARGENTINO DE

RACIONALIZACIÓN DE

MATERIALES. IRAM-AADL J20-

02: iluminación natural en edificios: condiciones generales y requisitos especiales. Bahia Blanca, 1970.

PATTINI, A. E. Recomendaciones de Niveles de Iluminación en Edificios no Residenciales: una comparación internacional. Averma, v. 4, n. 1, p. 5.07-5.12, 2000.

PATTINI, A. E. Eficiencia Lumínica de Dispositivos de Control y Difusión de la Luz Solar Aplicables a Ventanas en Aulas, en la Provincia de Mendoza, Argentina. San Miguel de Tucumán: Universidad Nacional de Tucumán, 2007.

114 Córica, L.; Lasagno, C.; Colombo, E.; Pattini, A. 
ROMBAUTS, P.; VANDEWYNGAERDE, H.;

MAGGETTO, G. Minimum Semicylindrical Illuminance and Modelling in Residential Area Lighting. Lighting Research and Technology, v. 21, n. 2, p. 49-55, jan. 1989.

ROMBAUTS, P. Modifying the Concept of Semi-Cylindrical Illuminance: further investigations on facial recognition. Belgium, 1995.

ROMBAUTS, P. Visual Comfort in Underground Working Environments. In: LUX-EUROPA LIGHTING CONFERENCE, 9., ReykjavikIjsland, 2001. Proceedings... Reykjavik-Ijsland, 2001.
SERVICIO METEOROLÓGICO NACIONAL ARGENTINO. Climatological Statistics: 20012010. Buenos Aires, 2011.

VOS, J. J. Disability Glare: a state of the art report. CIE Journal, v. 3, n. 2, p. 39-53, 1984.

WILDE, M.; MANZANO, E. La Visión de los Objetos Tridimensionales y la Relación Ec/Eh Como Caracterizadora de la Misma. n 1418. San Miguel de Tucumán: Laboratorio de Luminotecnia-Universidad Nacional de Tucumán, 1989.

\section{Lorena Córica}

Laboratorio de Ambiente Humano y Vivienda, Centro Científico Tecnológico | Consejo Nacional de Investigaciones Cientifícas y Técnicas | Av. Ruiz Leal s/n, Parque General San Martín | Mendoza - Argentina | 5500 | Tel.: +54 (261)-524-4345 | E-mail: Icorica@mendozaconicet.gob.ar

\section{Cecilia Lasagno}

Laboratorio de Ambiente Humano y Vivienda, Centro Científico Tecnológico | Consejo Nacional de Investigaciones Cientifícas y Técnicas | E-mail: clasagno@mendoza-conicet.gob.ar

\section{Elisa Colombo}

Departamento de Luminotecnia Luz y Visión, Facultad de Ciencias Exactas y Tecnologia | Universidad Nacional de Tucuman | Av. Independencia, 1.800 | San Miguel de Tucumán - Argentina | T4002BLR | Tel.: + (54) 436-4093 Ramal 316 | E-mail: ecolombo@herrera.unt.edu.ar

\section{Andrea Pattin}

Laboratorio de Ambiente Humano y Vivienda, Centro Científico Tecnológico | Consejo Nacional de Investigaciones Cientifícas y Técnicas | E-mail: apattini@mendoza-conicet.gob.ar

\section{Revista Ambiente Construído}

Associação Nacional de Tecnologia do Ambiente Construído

Av. Osvaldo Aranha, $99-3^{\circ}$ andar, Centro

Porto Alegre - RS - Brasil

$$
\text { CEP } 90035-190
$$

Telefone: +55 (51) 3308-4084

Fax: +55 (51) 3308-4054

www.seer.ufrgs.br/ambienteconstruido

E-mail: ambienteconstruido@ufrgs.br 